\title{
Relation of body composition to daily physical activity in free-living Japanese adult women
}

\author{
Jonghoon Park ${ }^{1 *}$, Kazuko Ishikawa-Takata ${ }^{1}$, Shigeho Tanaka ${ }^{1}$, Yuki Hikihara ${ }^{2}$, Kazunori Ohkawara ${ }^{1,3}$, \\ Shaw Watanabe ${ }^{1}$, Motohiko Miyachi ${ }^{1}$, Akemi Morita ${ }^{1}$, Naomi Aiba ${ }^{1}$ and Izumi Tabata ${ }^{1,4}$ \\ ${ }^{1}$ Health Promotion and Exercise Program, National Institute of Health and Nutrition, Tokyo, Japan \\ ${ }^{2}$ Faculty of Engineering, Chiba Institute of Technology, Narashino, Japan \\ ${ }^{3}$ Center for Human Nutrition, University of Colorado, Denver, CO, USA \\ ${ }^{4}$ Faculty of Sport and Health Science, Ritsumeikan University, Kusatsu, Shiga 525-8577, Japan
}

(Received 16 June 2010 - Revised 4 January 2011 - Accepted 14 February 2011 - First published online 17 May 2011)

\section{Abstract}

The objective of the present study was to investigate the relationship between the indices of body size such as BMI, fat-free mass index (FFMI, FFM/height ${ }^{2}$ ), fat mass index (FMI, FM/height ${ }^{2}$ ), and body fat percentage (\%BF), and physical activities assessed by the doublylabelled water (DLW) method and an accelerometer in free-living Japanese adult women. We conducted a cross-sectional study in 100 female subjects ranging in age from 31 to 69 years. Subjects were classified in quartiles of BMI, FFMI, FMI and \%BF. Daily walking steps and the duration of light to vigorous physical activity were simultaneously assessed by an accelerometer for the same period as the DLW experiment. Only physical activity-related energy expenditure (PAEE)/FFM and PAEE/body weight (BW) decreased in the highest quartile of BMI. Physical activity level, PAEE/FFM and PAEE/BW decreased in the highest quartile of FMI and \%BF, whereas they were not different among quartiles of FFMI. Daily walking steps and the duration of moderate- and vigorous-intensity physical activities decreased or tended to decrease in the highest quartile of FMI and \%BF, but did not differ among quartiles of FFMI and BMI. These results clearly showed that Japanese adult women with higher fat deposition obviously had a low level of physical activities assessed by both the DLW method and accelerometry, but those with larger BMI had lower PAEE/FFM and PAEE/BW only. Our data suggest that the relationship between obesity and daily physical activities should be discussed using not only BMI but also FMI or \%BF.

\section{Key words: Body composition: Physical activity: Doubly-labelled water: Accelerometry: Japanese adult women}

Obesity is caused by an imbalance between energy intake and energy expenditure. Obese individuals are often considered to be physically less active than normal-weight individuals. However, most cross-sectional studies using the doubly-labelled water (DLW) method, which is known to be the most accurate method of measuring energy expenditure in free-living conditions $^{(1,2)}$, have reported that physical activity level (PAL; the ratio of total energy expenditure(TEE):BMR) did not differ among BMI categories ${ }^{(3-6)}$. The reason for the lack of this association may be partly explained by differences in the distribution of fat-free mass (FFM) and fat mass (FM). PAL appears to be negatively associated with $\mathrm{FM}^{(7,8)}$, but not correlated with $\mathrm{FFM}^{(5)}$. However, these studies have only reported information on the association between PAL and either FM or FFM, which are not adjusted for body size, such as body height. To our knowledge, no information is available from thoroughly examining the relationship between BMI or body composition, i.e. FFM index (FFMI, FFM divided by height squared), FM index (FMI, FM divided by height squared) or body fat percentage (\%BF) and physical activity in adult women, particularly in Asian populations.

Recently, many cross-sectional studies on adult women in Western countries and Japan reported that BMI and \%BF were inversely associated with daily walking steps ${ }^{(9,10)}$. Furthermore, \%BF was negatively associated with the duration of vigorous-intensity physical activity assessed by accelerometry ${ }^{(11)}$. Therefore, not only physical activity-related energy expenditure (PAEE) but also the intensity of the physical activity or walking steps should be lower among adult women with higher body mass or fat deposition.

In the present study, we investigated the relationship between various indices of body size such as BMI, FFMI,

\footnotetext{
Abbreviations: \%BF, body fat percentage; BW, body weight; DHQ, diet history questionnaire; DMW, doubly-labelled water; FFM, fat-free mass; FFMI, fatfree mass index; FM, fat mass; FMI, fat mass index; METs, metabolic equivalents; PAEE, physical activity-related energy expenditure; PAL, physical activity level; SCOP, Saku Control Obesity Program; TEE, total energy expenditure.
} 
FMI and \%BF, and daily physical activities assessed by the DLW method and accelerometry in free-living Japanese adult women.

\section{Methods}

\section{Subjects}

Study participants were recruited through healthcare centres or at workplaces from various prefectures of the Kanto area (central Japan) and the Kyushu area (Western Japan), and from the Saku Control Obesity Program (SCOP). The details of SCOP are described elsewhere ${ }^{(12)}$. In each location, subjects were included according to the following criteria: (a) in good health; (b) not pregnant or breast-feeding; (c) BMI higher than $18.5 \mathrm{~kg} / \mathrm{m}^{2}$; (d) living in their home prefecture 2 weeks before and during the study; (e) not on a weight-loss or treatment diet; and (f) alcohol consumption less than $40 \mathrm{~g} / \mathrm{d}$. As a result, 100 female subjects aged 31 to 69 years participated in the present study. Daily physical activity was estimated over the $14 \mathrm{~d}$ study period in free-living conditions using the DLW method and accelerometry. Over the entire assessment period, subjects were carefully instructed to maintain their normal daily activities and eating patterns and to make no conscious effort to lose or gain weight.

\section{Procedures}

The experimental design is shown in Fig. 1. Participants completed two visits to study sites on day 0 and day 15 . On the day before the start of measuring physical activity (day 0), urine samples were collected early in the morning, $12 \mathrm{~h}$ or longer after the last meal (baseline urine sample), and body weight (BW) and height were measured. BMR was measured in the supine position and then the participants received a dose of DLW. On the day after the physical activity measurement (day 15), BW was measured and we then received back the urine samples, accelerometer and a self-administered diet history questionnaire (DHQ). The present study was conducted according to the guidelines laid down in the Declaration of Helsinki and all procedures involving human subjects were approved by the Ethical Committee of the National Institute of Health and Nutrition in Japan. All subjects gave their written informed consent before the commencement of the investigations.

\section{Anthropometric measures}

Anthropometric measures were obtained in the fasting state on the day before (day 0) and after the $14 \mathrm{~d}$ study period (day 15). BW was measured to the nearest $0 \cdot 1 \mathrm{~kg}$ and height to the nearest $0 \cdot 1 \mathrm{~cm}$, in individuals wearing the lightest clothing, with underwear and no shoes. BMI was calculated as BW (kg) divided by the square of body height $\left(\mathrm{m}^{2}\right)$.

\section{Diet history questionnaire}

The DHQ is a validated sixteen-page structured questionnaire that assesses dietary habits in the preceding 1-month period $^{(13)}$. Well-trained dietitians checked the DHQ to find omissions or errors and corrected them by asking questions of each participant. Details of the DHQ, methods of calculating nutrients and validity are given elsewhere ${ }^{(13)}$. We calculated the food quotient using the data from the DHQ to evaluate TEE.

\section{Doubly-labelled water}

After providing a baseline urine sample, a single dose of approximately $0.06 \mathrm{~g}^{2} \mathrm{H}_{2} \mathrm{O} / \mathrm{kg}$ BW (99.8 atom\%; Cambridge Isotope Laboratories, Andover, MA, USA) and $1.4 \mathrm{~g} \mathrm{H}_{2}{ }^{18} \mathrm{O} / \mathrm{kg}$ BW (10.0 atom\%; Taiyo Nippon Sanso, Tokyo, Japan) was given orally to each subject on day 0 . After dose administration, participants were asked to collect urine samples on day 1 (the day after the DLW dose) and on eight additional times during the study period at the same time of the day (Fig. 1). All urine samples except for the baseline one were collected by the participant either at home or their place of work, and the time of sampling was recorded. All samples were first stored by freezing at $-30^{\circ} \mathrm{C}$ in airtight parafilmwrapped containers, and then analysed in our laboratory.

\section{Gas analysis}

Gas samples for the isotope ratio mass spectrometer were prepared by equilibration of urine samples with a gas. The gas for equilibration of ${ }^{18} \mathrm{O}$ was $\mathrm{CO}_{2}$ and that for ${ }^{2} \mathrm{H}$ was $\mathrm{H}_{2}$. Pt catalyst was used for equilibration of ${ }^{2} \mathrm{H}$. The urine was analysed by a DELTA Plus isotope ratio mass spectrometer (Thermo Electron Corporation, Bremen, Germany). Each sample and the corresponding reference were analysed in duplicate.

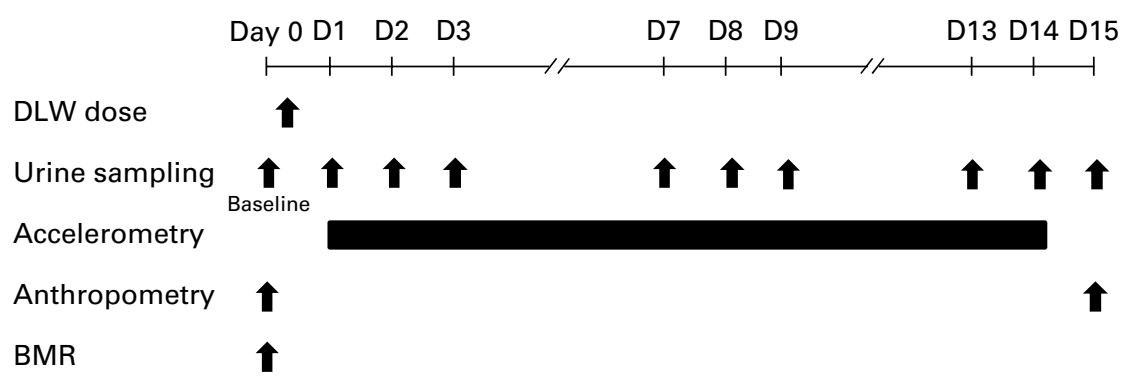

Fig. 1. Schematic representation of the experimental design. On day 0 , the ${ }^{2} \mathrm{H}_{2}{ }^{18} \mathrm{O}$ (doubly-labelled water; DLW) dose was given orally to each subject after collecting a baseline urine sample and performing the BMR and anthropometric measurements. 
The average standard deviations through the analyses were $0 \cdot 5 \%$ for ${ }^{2} \mathrm{H}$ and $0.03 \%$ for ${ }^{18} \mathrm{O}$.

\section{Calculations of total energy expenditure and body composition}

The ${ }^{2} \mathrm{H}$ and ${ }^{18} \mathrm{O}$ zero-time intercepts and elimination rates $\left(\mathrm{k}_{\mathrm{H}}\right.$ and $\mathrm{k}_{\mathrm{O}}$ ) were calculated by using a least-squares linear regression on the natural logarithm of the isotope concentration as a function of the elapsed time from dose administration. The zero-time intercepts were used to determine the isotope pool sizes. Total body water (TBW) was calculated from the mean value of the isotope pool size of ${ }^{2} \mathrm{H}$ divided by 1.041 and that of ${ }^{18} \mathrm{O}$ divided by $1 \cdot 007$. FFM was calculated assuming a FFM hydration of $0.732^{(14)}$. FM was calculated as $\mathrm{BW}$ minus FFM and \%BF was then computed from BW and FFM. The TEE (kJ/d) calculation was performed using a modification of Weir's formula ${ }^{(15)}$ based on the $\mathrm{CO}_{2}$ production rate $\left(\mathrm{rCO}_{2}\right)$ and respiratory quotient. $\mathrm{rCO}_{2}$ was calculated as follows: $\quad \mathrm{rCO}_{2}=0.4554 \times \mathrm{TBW} \times\left(1.007 \mathrm{ko}-1.041 \mathrm{k}_{\mathrm{H}}\right)$. The food quotient calculated from DHQ was used instead of the respiratory quotient. This assumes that under conditions of perfect nutrient balance the food quotient must equal the respiratory quotient ${ }^{(16,17)}$. PAL was estimated by dividing TEE by BMR. PAEE was calculated as $0.9 \times$ TEE - BMR, assuming the thermic effect of food was $10 \% \mathrm{of} \mathrm{TEE}^{(18)}$.

\section{$B M R$}

BMR was measured in the supine position in the early morning $12 \mathrm{~h}$ or longer after the last meal, as described previously $^{(19)}$. The measurement was performed using a Douglas bag for $10 \mathrm{~min} \times 2$ with $1 \mathrm{~min}$ of intermission. After the expired air was sampled, the $\mathrm{O}_{2}$ and $\mathrm{CO}_{2}$ concentrations were measured using a gas analyser (Arco System, AR-1, Kashiwa, Japan for the participants from the SCOP study, or Arco System, ARCO-1000, Kashiwa, Japan, for the rest of the participants) and the volume of expired air was measured with a certified dry gas meter (DC-5; Shinagawa, Tokyo, Japan). BMR was estimated from $\mathrm{O}_{2}$ consumption and $\mathrm{CO}_{2}$ production using Weir's equation ${ }^{(15)}$.

\section{Accelerometry}

The Lifecorder EX (Suzuken Co., Ltd, Nagoya, Japan) is a uniaxial accelerometer widely used in many countries due to its reasonable cost and reliable validity for measuring metabolic equivalents (METs) and step counts ${ }^{(20-22)}$. In the present study, the Lifecorder EX was attached on the left side of the waist at the midline of the left thigh. The movement data are categorised into eleven activity levels (0, 0.5, and 1 to 9). We applied METs for each activity level according to the study of Kumahara et al., and the intensity of activity was divided into light ( $<3$ METs), moderate $(\geq 3$ and $<6$ METs) and vigorous $(\geq 6 \text { METs })^{(20)}$.

\section{Statistics}

All values are presented as mean values and standard deviations. BMI was calculated as BW (measured before DLW dose) divided by height squared. FFMI and FMI were calculated as FFM and FM divided by height squared, respectively. Subjects were classified by quartiles of BMI, FFMI, FMI and $\% \mathrm{BF}$. Homoscedasticity or homogeneity of variances was examined using Levene's test. Because some variables in physical characteristics did not follow a normal distribution, the non-parametric test of Kruskal-Wallis analysis was used to compare the variables in physical characteristics among quartiles, and the Mann-Whitney $U$ test was used for multiple comparisons. In variables that were normally distributed, one-way ANOVA was used to compare the variables among quartiles and Fisher's least square difference was used as a post hoc test for multiple comparisons. The associations between physical activities and body size or composition were examined by linear regression analysis. In one-way ANOVA, post hoc tests and Kruskal-Wallis tests, differences were considered to be statistically significant if the $P$ value was less than 0.05; using the Mann-Whitney $U$ test, differences were deemed significant at $P<0.0125$ (modification using Bonferroni's inequality). All statistical treatments were done using SPSS for Windows (version 16.0 J; SPSS Inc., Chicago, IL, USA).

\section{Results}

Of the total 100 women studied, the proportion of normalweight (BMI $\geq 18.5$ to $<25 \mathrm{~kg} / \mathrm{m}^{2}$ ) and overweight participants (BMI $\geq 25 \mathrm{~kg} / \mathrm{m}^{2}$ ) was 76 and $24 \%$, respectively. The mean age of the subjects was $51 \cdot 8$ (SD 11.2; range 31-69) years. The mean BW and BMI were 57.4 (SD 12.2; range $41.7-109 \cdot 7$ ) $\mathrm{kg}$ and 23.5 (SD 4.4 ; range $18 \cdot 8-40 \cdot 0) \mathrm{kg} / \mathrm{m}^{2}$, respectively. BW did not change during the study (change of $\mathrm{BW} 0.02$ (SD 0.7 ) kg; $P=0.987$ ). The range of $\mathrm{PAL}$ was $1 \cdot 36-2 \cdot 52$, with a mean value of 1.88 .

Physical characteristics and physical activity variables among quartiles of BMI, FFMI, FMI and \%BF are shown in Tables 1-4, respectively. Among the physical characteristics, age and height were not significantly different among quartiles. BMI increased linearly with FMI ( $r$ 0.943) and \%BF $(r$ 0.749), whereas FFM increased in the 4th quartiles of FMI and $\% \mathrm{BF}$ (Tables 3 and 4 ).

Of energy expenditure components, TEE/BW decreased linearly with BMI, FMI and \%BF. On the other hand, TEE/BW decreased only in the 4th quartile of FFMI (Table 2). PAEE/ FFM and PAEE/BW decreased in the 4th quartile of BMI, but PAL did not differ among quartiles (Table 1). Among FFMI quartiles, there were no significant differences among PAL, PAEE/FFM and PAEE/BW. However, among FMI quartiles, all PAL, PAEE/FFM and PAEE/BW decreased in the 4th quartile. Among \%BF quartiles, PAL and PAEE/FFM were significantly lower in the 3rd and 4th quartiles than in the 2nd quartile, whereas PAEE/BW decreased from the 3rd quartile. Fig. 2 shows that PAL was negatively associated with FMI, but not with BMI and FFMI (Fig. 2). PAEE/FFM and PAEE/BW were 
Table 1. Participant characteristics, energy expenditure components and physical activity variables by BMI grouping

(Mean values and standard deviations)

\begin{tabular}{|c|c|c|c|c|c|c|c|c|c|c|}
\hline \multirow[t]{2}{*}{ BMI $\left(\mathrm{kg} / \mathrm{m}^{2}\right)$ quartiles $\| \ldots$} & \multicolumn{2}{|c|}{ 1st (18.6-20.4) } & \multicolumn{2}{|c|}{ 2nd (20.5-22.1) } & \multicolumn{2}{|c|}{ 3rd (22.3-24.7) } & \multicolumn{2}{|c|}{ 4th $(24 \cdot 7-40 \cdot 0)$} & \multirow[b]{2}{*}{$P$ (ANOVA) } & \multirow[b]{2}{*}{$r$} \\
\hline & Mean & SD & Mean & SD & Mean & SD & Mean & SD & & \\
\hline \multicolumn{11}{|l|}{ Physical characteristics } \\
\hline Age (years) & $49 \cdot 7$ & 11.9 & 51.4 & $11 \cdot 8$ & 53.9 & 11.9 & 52.4 & 9.4 & 0.630 & 0.038 \\
\hline Height $(\mathrm{m})$ & 1.55 & 0.04 & 1.56 & 0.06 & 1.56 & 0.04 & 1.56 & 0.06 & 0.890 & 0.133 \\
\hline Weight $(\mathrm{kg})$ ? & $47 \cdot 1$ & $3 \cdot 1$ & $52 \cdot 1+\dagger$ & $4 \cdot 2$ & 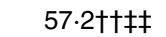 & 3.3 & 73.0††‡§§ & $13 \cdot 4$ & $<0.001$ & $0.948^{\star * \star}$ \\
\hline BMI $\left(\mathrm{kg} / \mathrm{m}^{2}\right)$ & 19.5 & 0.6 & $21 \cdot 3+\dagger$ & 0.5 & $23.5+† \neq \ddagger$ & 0.9 & $29 \cdot 8 \dagger \dagger \ddagger \ddagger \S \S$ & 3.9 & $<0.001$ & 1 \\
\hline$\% B F q$ & 28.9 & $5 \cdot 1$ & $32 \cdot 3$ & $4 \cdot 3$ & $36 \cdot 0 \dagger † \ddagger ~$ & $5 \cdot 0$ & $42 \cdot 0 \dagger+\ddagger £ \S \S$ & 4.6 & $<0.001$ & $0.747^{\star \star \star}$ \\
\hline FFM (kg)ף & 33.5 & 2.5 & $35 \cdot 7$ & 3.6 & $36 \cdot 3+\dagger$ & 3.8 & $42 \cdot 2 \dagger \dagger \ddagger \ddagger \S \S$ & $6 \cdot 7$ & $<0.001$ & $0.743^{\star \star \star}$ \\
\hline $\mathrm{FM}(\mathrm{kg}) \boldsymbol{\eta}$ & $13 \cdot 7$ & $2 \cdot 8$ & $16 \cdot 9+\dagger$ & $2 \cdot 7$ & 20.6††㧊 & 3.3 & $30.5 \dagger \dagger \neq \ddagger \S \S$ & $7 \cdot 7$ & $<0.001$ & $0.930^{\star * \star}$ \\
\hline \multicolumn{11}{|l|}{ Energy expenditure } \\
\hline TEE $(\mathrm{kJ} / \mathrm{d})$ & 8441 & 1149 & 8534 & 883 & $9333 \dagger \ddagger$ & 1244 & 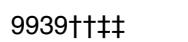 & 1523 & $<0.001$ & $0.527^{\star \star \star}$ \\
\hline TEE/BW (kJ/d per kg) & $179 \cdot 8$ & $27 \cdot 1$ & $164 \cdot 7 \dagger$ & $21 \cdot 2$ & $163.5 \dagger$ & 23.0 & $138 \cdot 1 \dagger \dagger \neq \S \S$ & 20.4 & $<0.001$ & $-0.588^{\star \star \star}$ \\
\hline BMR $(\mathrm{kJ} / \mathrm{d})$ & 4492 & 351 & 4604 & 462 & 4777 & 588 & $5558+† \neq \neq \S \S$ & 892 & $<0.001$ & $0.725^{\star \star \star}$ \\
\hline PAL & $1 \cdot 88$ & 0.23 & 1.85 & 0.22 & 1.97 & 0.27 & 1.80 & $0 \cdot 18$ & 0.065 & -0.187 \\
\hline PAEE $(\mathrm{kJ} / \mathrm{d})$ & 3105 & 913 & 3077 & 747 & 3623 & 1069 & 3387 & 886 & 0.099 & 0.120 \\
\hline PAEE/FFM (kJ/d per kg) & 92.4 & $24 \cdot 8$ & 86.8 & 21.8 & $100 \cdot 7 \ddagger$ & $30 \cdot 6$ & $81.3 \S$ & $20 \cdot 3$ & 0.040 & $-0.207^{\star}$ \\
\hline PAEE/BW (kJ/d per kg) & $66 \cdot 2$ & $20 \cdot 6$ & $59 \cdot 7$ & $16 \cdot 0$ & 63.8 & $19 \cdot 7$ & $47.5 \dagger \dagger \neq \S \S$ & $13 \cdot 1$ & 0.001 & $-0.403^{\star \star *}$ \\
\hline \multicolumn{11}{|l|}{ Accelerometer } \\
\hline Step counts (per d) & 8994 & 2151 & 8872 & 2619 & 8624 & 2729 & 7808 & 3402 & 0.427 & $-0.286^{* *}$ \\
\hline Light $(<3 \mathrm{METs})(\mathrm{min} / \mathrm{d})$ & $57 \cdot 0$ & $15 \cdot 8$ & 58.4 & 23.0 & $62 \cdot 0$ & $24 \cdot 8$ & $55 \cdot 0$ & $20 \cdot 3$ & 0.691 & -0.107 \\
\hline Moderate $(\geq 3$ and $<6$ METs) $(\mathrm{min} / \mathrm{d})$ & $28 \cdot 8$ & $12 \cdot 0$ & $27 \cdot 1$ & $13 \cdot 8$ & $23 \cdot 3$ & $10 \cdot 2$ & $21 \cdot 0$ & $13 \cdot 8$ & $0 \cdot 122$ & $-0.316^{\star \star}$ \\
\hline Vigorous ( $\geq 6 \mathrm{METs})(\mathrm{min} / \mathrm{d})$ & 3.7 & 3.4 & 3.0 & $2 \cdot 9$ & $2 \cdot 7$ & $2 \cdot 7$ & $2 \cdot 0$ & $2 \cdot 7$ & 0.246 & $-0.239^{\star}$ \\
\hline
\end{tabular}

\%BF, body fat percentage; FFM, fat-free mass; FM, fat mass; TEE, total energy expenditure; BW, body weight; PAL, physical activity level (=TEE/BMR); PAEE, physical activity energy expenditure ( $=0.9 \mathrm{TEE}-\mathrm{BMR})$; METs, metabolic equivalents.

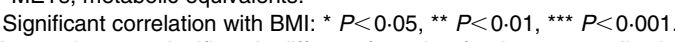

Mean value was significantly different from that for the 1 st quartile: $\dagger P<0.05$, t† $P<0.01$

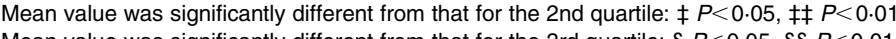

Mean value was significantly different from that for the 3rd quartle. $\S P<0.05$, $\S \S P<0.01$.

" Because some variables in physical characteristics did not follow a normal distribution, Kruskal-Wallis analysis was used to compare the variables among quartiles, and the Mann-Whitney $U$ test was used for multiple 
Table 2. Participant characteristics, energy expenditure components and physical activity variables by fat-free mass index (FFMI) grouping (Mean values and standard deviations)

\begin{tabular}{|c|c|c|c|c|c|c|c|c|c|c|}
\hline \multirow[t]{2}{*}{ FFMI quartiles $\| \ldots$} & \multicolumn{2}{|c|}{ 1st $(12 \cdot 2-13 \cdot 8)$} & \multicolumn{2}{|c|}{ 2nd $(13 \cdot 8-14 \cdot 6)$} & \multicolumn{2}{|c|}{ 3rd $(14 \cdot 7-15 \cdot 6)$} & \multicolumn{2}{|c|}{ 4th $(15 \cdot 7-21 \cdot 6)$} & \multirow[b]{2}{*}{$P($ ANOVA $)$} & \multirow[b]{2}{*}{$r$} \\
\hline & Mean & SD & Mean & SD & Mean & SD & Mean & SD & & \\
\hline \multicolumn{11}{|l|}{ Physical characteristics } \\
\hline Age (years) & 48.5 & $12 \cdot 9$ & $55 \cdot 6$ & $10 \cdot 5$ & $54 \cdot 0$ & $10 \cdot 9$ & $49 \cdot 1$ & $9 \cdot 1$ & 0.054 & -0.026 \\
\hline Height $(m)$ & 1.56 & 0.05 & 1.56 & 0.05 & 1.55 & 0.06 & 1.57 & 0.05 & 0.587 & 0.093 \\
\hline Weight $(\mathrm{kg})$ ฯ & $50 \cdot 1$ & 4.4 & $52 \cdot 0$ & 4.5 & $56 \cdot 2 \dagger \dagger$ & $7 \cdot 7$ & $71 \cdot 1 \dagger \dagger \ddagger £ \S \S$ & $15 \cdot 1$ & $<0.001$ & $0 \cdot 753^{\star \star \star}$ \\
\hline BMI $\left(\mathrm{kg} / \mathrm{m}^{2}\right)$ व & $20 \cdot 6$ & 1.4 & $21 \cdot 6$ & $2 \cdot 1$ & $23 \cdot 3+\dagger$ & $2 \cdot 6$ & $28 \cdot 7 \dagger \dagger \neq £ \S \S$ & $5 \cdot 2$ & $<0.001$ & $0.794^{\star \star \star}$ \\
\hline$\% B F q$ & 34.9 & 4.0 & $32 \cdot 8$ & $6 \cdot 2$ & 33.9 & $7 \cdot 4$ & 37.6 & 8.3 & 0.045 & $0.247^{\star}$ \\
\hline FFM (kg)ף & $32 \cdot 2$ & $2 \cdot 0$ & $34.6+\dagger$ & $2 \cdot 2$ & 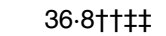 & $2 \cdot 8$ & $44 \cdot 0 \dagger † \ddagger \ddagger \S \S$ & 4.9 & $<0.001$ & $0.890^{\star \star \star}$ \\
\hline$F M(k g)$ ๆ & $17 \cdot 6$ & 3.2 & $17 \cdot 2$ & 4.5 & 19.5 & $6 \cdot 4$ & $27 \cdot 3+\dagger \neq £ \S \S$ & $10 \cdot 5$ & $<0.001$ & $0.581^{\star \star \star}$ \\
\hline FFMI $\left(\mathrm{kg} / \mathrm{m}^{2}\right)$ & $13 \cdot 3$ & 0.4 & $14 \cdot 3$ & 0.3 & $15 \cdot 2$ & 0.3 & $17 \cdot 8$ & 1.5 & $<0.001$ & 1 \\
\hline \multicolumn{11}{|l|}{ Energy expenditure } \\
\hline TEE $(k J / d)$ & 8017 & 891 & 8676 & 932 & $9306+\dagger$ & 1100 & $10248 \dagger † \ddagger \ddagger \S \S$ & 1358 & $<0.001$ & $0.626^{\star \star \star}$ \\
\hline TEE/BW (kJ/d per kg) & $160 \cdot 9$ & $20 \cdot 2$ & $167 \cdot 6$ & $20 \cdot 2$ & $169 \cdot 3$ & $35 \cdot 2$ & $148 \cdot 4 \ddagger \S$ & $26 \cdot 8$ & 0.025 & $-0.262^{\star *}$ \\
\hline $\operatorname{BMR}(\mathrm{kJ} / \mathrm{d})$ & 4391 & 444 & 4582 & 423 & 4871††‡ & 533 & $5587 \dagger † \neq \pm \S \S$ & 826 & $<0.001$ & $0.708^{\star \star \star}$ \\
\hline PAL & 1.83 & 0.18 & 1.91 & 0.24 & 1.92 & 0.29 & $1 \cdot 85$ & 0.20 & 0.484 & -0.064 \\
\hline PAEE $(k J / d)$ & 2824 & 659 & 3226 & 841 & $3505 \dagger$ & 1090 & $3636+\dagger$ & 890 & 0.011 & $0.263^{\star \star}$ \\
\hline PAEE/FFM (kJ/d per kg) & 88.0 & 21.9 & 93.4 & 24.5 & $96 \cdot 3$ & $31 \cdot 0$ & 83.6 & $22 \cdot 6$ & 0.368 & -0.151 \\
\hline PAEE/BW (kJ/d per kg) & $56 \cdot 6$ & $13 \cdot 1$ & $62 \cdot 4$ & $17 \cdot 1$ & 64.5 & $24 \cdot 7$ & 53.6 & $17 \cdot 3$ & $0 \cdot 182$ & -0.157 \\
\hline \multicolumn{11}{|l|}{ Accelerometer } \\
\hline Step counts (per d) & 8589 & 2592 & 8914 & 2437 & 8267 & 2635 & 8528 & 3403 & 0.878 & -0.159 \\
\hline Light (<3 METs) $(\mathrm{min} / \mathrm{d})$ & $53 \cdot 6$ & $20 \cdot 4$ & $59 \cdot 1$ & $17 \cdot 2$ & $55 \cdot 7$ & 18.9 & $64 \cdot 1$ & $26 \cdot 5$ & 0.320 & 0.040 \\
\hline Moderate $(\geq 3$ and $<6$ METs) $(\mathrm{min} / \mathrm{d})$ & $28 \cdot 0$ & $15 \cdot 2$ & $27 \cdot 3$ & $10 \cdot 4$ & 23.9 & $12 \cdot 0$ & $21 \cdot 1$ & $12 \cdot 3$ & 0.187 & $-0.300^{\star *}$ \\
\hline Vigorous ( $\geq 6 \mathrm{METs})(\mathrm{min} / \mathrm{d})$ & 3.4 & 3.0 & 2.6 & $2 \cdot 8$ & $3 \cdot 1$ & 3.6 & $2 \cdot 3$ & $2 \cdot 3$ & 0.513 & -0.108 \\
\hline
\end{tabular}

$\% B F$, body fat percentage; FFM, fat-free mass; FM, fat mass; TEE, total energy expenditure; BW, body weight; PAL, physical activity level ( = TEE/BMR); PAEE, physical activity energy expenditure ( $=0.9 T E E-B M R) ;$ METs, metabolic equivalents.

* Significant correlation with FFMI: * $P<0.05,{ }^{* *} P<0.01,{ }^{* * *} P<0.001$

Mean value was significantly different from that for the 1st quartile: $\dagger P<0.05$, $\dagger \dagger P<0.01$

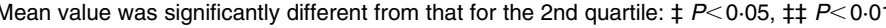

Mean value was significantly different from that for the 3rd quartile: $\S P<0.05$, $\S \S P<0.01$

$\|$ Subjects were categorised by quartile. There are twenty-five subjects in each quartile.

\Because some variables in physical characteristics did not follow a normal distribution, Kruskal-Wallis analysis was used to compare the variables among quartiles, and the Mann-Whitney $U$ test was used for multiple comparisons. 
Table 3. Participant characteristics, energy expenditure components and physical activity variables by fat mass index (FMI) grouping

(Mean values and standard deviations)

\begin{tabular}{|c|c|c|c|c|c|c|c|c|c|c|}
\hline \multirow[t]{2}{*}{ FMI quartiles $\| \ldots$} & \multicolumn{2}{|c|}{ 1st (2.94-6.39) } & \multicolumn{2}{|c|}{ 2nd (6.49-7.52) } & \multicolumn{2}{|c|}{ 3rd (7.55-9.73) } & \multicolumn{2}{|c|}{ 4th $(9 \cdot 82-19 \cdot 49)$} & \multirow[b]{2}{*}{$P($ ANOVA $)$} & \multirow[b]{2}{*}{$r$} \\
\hline & Mean & SD & Mean & SD & Mean & SD & Mean & SD & & \\
\hline \multicolumn{11}{|l|}{ Physical characteristics } \\
\hline Age (years) & $49 \cdot 9$ & $10 \cdot 9$ & $52 \cdot 4$ & $12 \cdot 2$ & 51.4 & $11 \cdot 6$ & 53.5 & $10 \cdot 3$ & 0.713 & 0.085 \\
\hline Height $(m)$ & 1.56 & 0.05 & 1.56 & 0.05 & 1.56 & 0.05 & 1.56 & 0.06 & 0.921 & 0.138 \\
\hline Weight $(\mathrm{kg})$ q & 48.3 & 4.5 & $51 \cdot 7$ & 4.5 & 56.7††キ‡ & 4.4 & $72 \cdot 8 \dagger † \ddagger \ddagger \S \S$ & 13.5 & $<0.001$ & $0.897^{\star \star \star}$ \\
\hline BMI $\left(\mathrm{kg} / \mathrm{m}^{2}\right)$ ๆ & $19 \cdot 9$ & 1.2 & $21 \cdot 3+\dagger$ & 1.2 & 23·2†††キ & $1 \cdot 7$ & 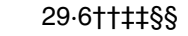 & $4 \cdot 2$ & $<0.001$ & $0.943^{\star \star *}$ \\
\hline \%BFq & $26 \cdot 4$ & 4.2 & $32 \cdot 9+\dagger$ & 1.5 & $37 \cdot 1 \dagger † \ddagger \ddagger$ & 1.7 & $42 \cdot 9 \dagger \dagger \neq \pm \S \S$ & 3.9 & $<0.001$ & $0.916^{\star \star \star}$ \\
\hline FFM (kg)ף & $35 \cdot 6$ & 3.9 & 34.9 & 4.0 & 35.7 & 3.3 & $41.5 \dagger † \ddagger ‡ \S \S$ & $7 \cdot 1$ & 0.001 & $0.565^{\star \star \star}$ \\
\hline $\mathrm{FM}(\mathrm{kg}) \boldsymbol{\eta}$ & $12 \cdot 8$ & $2 \cdot 4$ & $17 \cdot 0+\dagger$ & 1.3 & 21.0††立 & 1.7 & $30 \cdot 9 \dagger \dagger \neq \pm \S \S$ & $7 \cdot 2$ & $<0.001$ & $0.982^{\star \star \star}$ \\
\hline FMI (range) $\left(\mathrm{kg} / \mathrm{m}^{2}\right)$ & $5 \cdot 3$ & 0.9 & $7 \cdot 0$ & 0.3 & $8 \cdot 6$ & 0.7 & $12 \cdot 6$ & $2 \cdot 3$ & $<0.001$ & 1 \\
\hline \multicolumn{11}{|l|}{ Energy expenditure } \\
\hline TEE (kJ/d) & 8810 & 1097 & 8782 & 1258 & 9049 & 1346 & 9607 & 1576 & 0.110 & $0.352^{\star \star \star}$ \\
\hline TEE/BW (kJ/d per kg) & 183.4 & $25 \cdot 4$ & $170 \cdot 0 \dagger$ & $20 \cdot 7$ & $159.4 \dagger \dagger$ & $17 \cdot 2$ & 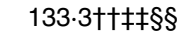 & $16 \cdot 7$ & $<0.001$ & $-0.696^{\star \star *}$ \\
\hline BMR $(\mathrm{kJ} / \mathrm{d})$ & 4586 & 375 & 4584 & 457 & 4760 & 559 & $5503+† \ddagger \ddagger \S \S$ & 971 & $<0.001$ & $0.610^{\star \star \star}$ \\
\hline PAL & 1.91 & 0.22 & 1.93 & 0.28 & 1.91 & 0.21 & $1 \cdot 76+\ddagger \S$ & 0.19 & 0.036 & $-0.254^{\star}$ \\
\hline PAEE (kJ/d) & 3343 & 847 & 3320 & 1082 & 3384 & 914 & 3143 & 876 & 0.827 & -0.017 \\
\hline PAEE/FFM (kJ/d per kg) & $94 \cdot 3$ & $23 \cdot 6$ & 95.9 & $31 \cdot 3$ & $94 \cdot 3$ & $21 \cdot 1$ & $76 \cdot 8 \dagger \ddagger \ddagger \S$ & $20 \cdot 4$ & 0.024 & $-0.258^{\star \star}$ \\
\hline PAEE/BW (kJ/d per kg) & $69 \cdot 6$ & $19 \cdot 0$ & $64 \cdot 2$ & 19.5 & $59 \cdot 4 \dagger$ & $14 \cdot 0$ & $43 \cdot 9 \dagger † \ddagger \ddagger \S \S$ & $11 \cdot 7$ & $<0.001$ & $-0.502^{\star \star *}$ \\
\hline \multicolumn{11}{|l|}{ Accelerometer } \\
\hline Step counts (per d) & 8508 & 2034 & 9724 & 2154 & 8866 & 3387 & $7200 \ddagger \ddagger \S$ & 2777 & 0.011 & $-0.293^{\star \star}$ \\
\hline Light (<3 METs) $(\mathrm{min} / \mathrm{d})$ & 56.5 & $17 \cdot 0$ & 63.0 & $21 \cdot 2$ & 61.3 & $26 \cdot 5$ & $51 \cdot 7$ & $17 \cdot 8$ & 0.224 & -0.156 \\
\hline Moderate $(\geq 3$ and $<6$ METs) $(\mathrm{min} / \mathrm{d})$ & 24.9 & $9 \cdot 7$ & $30 \cdot 3$ & $13 \cdot 2$ & $25 \cdot 7$ & 14.6 & 19.3ł‡ & $11 \cdot 0$ & 0.021 & $-0 \cdot 265^{\star \star}$ \\
\hline Vigorous ( $\geq 6$ METs) $(\mathrm{min} / \mathrm{d})$ & $3 \cdot 8$ & 3.5 & 3.5 & $3 \cdot 0$ & $2 \cdot 3$ & $2 \cdot 1$ & $1 \cdot 8 \dagger \ddagger$ & $2 \cdot 7$ & 0.042 & $-0.282^{\star \star}$ \\
\hline
\end{tabular}

$\% \mathrm{BF}$, body fat percentage; FFM, fat-free mass; FM, fat mass; TEE, total energy expenditure; BW, body weight; PAL, physical activity level (=TEE/BMR); PAEE, physical activity energy expenditure (=0.9TEE - BMR);

* Significant correlation with FMI: * $P<0.05,{ }^{* *} P<0.01,{ }^{* * *} P<0.001$

Mean value was significantly different from that for the 1 st quartile: $+P<0.05$, t† $P<0.01$

Mean value was significantly different from that for the 2 nd quartile: $\neq P<0.05$, t+ $P<0.01$

Mean value was significantly different from that for the 3rd quartile: $\S P<0.05$, $\S \S P<0.01$

Subjects were categorised by quartile. There are twenty-five subjects in each quartile.

1) Because some
comparisons. 
Table 4. Participant characteristics, energy expenditure components and physical activity variables by body fat percentage (\%BF) grouping (Mean values and standard deviations)

\begin{tabular}{|c|c|c|c|c|c|c|c|c|c|c|}
\hline \multirow[t]{2}{*}{$\% B F$ quartiles $\| \ldots$} & \multicolumn{2}{|c|}{ 1st $(15 \cdot 9-31 \cdot 0)$} & \multicolumn{2}{|c|}{ 2nd (31.4-34.5) } & \multicolumn{2}{|c|}{ 3rd (34.6-38.8) } & \multicolumn{2}{|c|}{ 4th $(39 \cdot 1-54 \cdot 3)$} & \multirow[b]{2}{*}{$P$ (ANOVA) } & \multirow[b]{2}{*}{$r$} \\
\hline & Mean & SD & Mean & SD & Mean & SD & Mean & SD & & \\
\hline \multicolumn{11}{|l|}{ Physical characteristics } \\
\hline Age (years) & $48 \cdot 7$ & $10 \cdot 6$ & 53.8 & $12 \cdot 3$ & $50 \cdot 3$ & 11.3 & 53.8 & $10 \cdot 2$ & 0.596 & 0.138 \\
\hline Height $(m)$ & 1.56 & 0.06 & 1.55 & 0.04 & 1.56 & 0.05 & 1.57 & 0.06 & 0.839 & 0.112 \\
\hline Weight $(\mathrm{kg}) \uparrow$ & 49.0 & $5 \cdot 4$ & $53.4 \dagger$ & $6 \cdot 5$ & $54 \cdot 8+\dagger$ & $4 \cdot 3$ & $72 \cdot 3+† \ddagger \ddagger \S \S$ & 13.9 & $<0.001$ & $0.710^{\star \star \star}$ \\
\hline BMI $\left(\mathrm{kg} / \mathrm{m}^{2}\right)$ ๆ & $20 \cdot 1$ & 1.3 & $22 \cdot 1+\dagger$ & $2 \cdot 2$ & $22 \cdot 6+\dagger$ & $2 \cdot 0$ & $29 \cdot 3+† \ddagger \ddagger \S \S$ & 4.5 & $<0.001$ & $0.749^{\star \star \star}$ \\
\hline$\%$ BFq & $26 \cdot 2$ & $4 \cdot 1$ & $32 \cdot 7 \dagger \dagger$ & 0.9 & 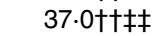 & 1.2 & $43 \cdot 2 \dagger \dagger \ddagger \pm \S \S$ & 3.4 & $<0.001$ & 1 \\
\hline FFM $(\mathrm{kg})$ ฯ & $36 \cdot 1$ & $4 \cdot 2$ & 36.0 & 4.5 & 34.5 & $2 \cdot 6$ & $41.0 \neq \S \S$ & $7 \cdot 2$ & 0.005 & $0.278^{\star *}$ \\
\hline $\mathrm{FM}(\mathrm{kg}) \uparrow$ & $12 \cdot 9$ & $2 \cdot 7$ & $17.5+\dagger$ & $2 \cdot 4$ & $20 \cdot 3 † \dagger \ddagger$ & 1.8 & $30 \cdot 9+† \ddagger \ddagger \S \S$ & $7 \cdot 2$ & $<0.001$ & $0.889^{\star \star \star}$ \\
\hline \multicolumn{11}{|l|}{ Energy expenditure } \\
\hline TEE $(\mathrm{kJ} / \mathrm{d})$ & 8845 & 1091 & 9326 & 1375 & 8600 & 1090 & 9477 & 1657 & 0.074 & 0.122 \\
\hline TEE/BW ( $\mathrm{kJ} / \mathrm{d}$ per $\mathrm{kg})$ & $182 \cdot 1$ & 26.9 & $175 \cdot 0$ & $19 \cdot 4$ & 156.6††キ‡ & $13 \cdot 1$ & $132 \cdot 4 \dagger † \ddagger £ \S$ & 15.5 & $<0.001$ & $-0.725^{\star * \star}$ \\
\hline BMR (kJ/d) & 4640 & 372 & 4727 & 530 & 4680 & 556 & $5385+† \ddagger \ddagger \S \S$ & 1041 & $<0.001$ & $0.368^{\star \star \star}$ \\
\hline PAL & 1.90 & 0.22 & 1.98 & 0.26 & $1.85 \ddagger$ & 0.22 & $1 \cdot 78 \neq \ddagger$ & 0.19 & 0.013 & $-0.243^{\star}$ \\
\hline PAEE $(k J / d)$ & 3321 & 861 & 3666 & 1072 & 3059 & 806 & 3144 & 872 & 0.099 & -0.124 \\
\hline PAEE/FFM (kJ/d per kg) & 92.5 & 24.5 & $102 \cdot 6$ & $29 \cdot 6$ & $88 \cdot 2 \ddagger$ & $20 \cdot 6$ & 77.9ł‡ & $20 \cdot 6$ & 0.006 & $-0.244^{\star}$ \\
\hline PAEE/BW (kJ/d per kg) & 68.5 & $19 \cdot 8$ & $68 \cdot 7$ & $18 \cdot 1$ & $55.5 \dagger † \ddagger$ & $12 \cdot 8$ & $44.4 \dagger † \ddagger \ddagger \S$ & $12 \cdot 0$ & $<0.001$ & $-0.515^{\star \star \star}$ \\
\hline \multicolumn{11}{|l|}{ Accelerometer } \\
\hline Step counts (per d) & 8675 & 2082 & 9449 & 2173 & 9067 & 3288 & $7107 \dagger \ddagger \ddagger \S$ & 2869 & 0.013 & $-0.293^{\star \star}$ \\
\hline Light $(<3$ METs) $(\mathrm{min} / \mathrm{d})$ & 58.0 & $16 \cdot 2$ & 64.9 & $23 \cdot 1$ & $59 \cdot 2$ & $24 \cdot 6$ & $50 \cdot 4$ & $18 \cdot 1$ & 0.113 & $-0.168^{\star}$ \\
\hline Moderate $(\geq 3$ and $<6 \mathrm{METs})(\mathrm{min} / \mathrm{d})$ & $25 \cdot 7$ & $10 \cdot 2$ & $26 \cdot 4$ & $11 \cdot 2$ & 28.7 & $15 \cdot 7$ & $19 \cdot 4$ & 11.8 & 0.057 & -0.154 \\
\hline Vigorous ( $\geq 6 \mathrm{METs})(\mathrm{min} / \mathrm{d})$ & 3.4 & 3.4 & 3.9 & 3.0 & $2 \cdot 3$ & $2 \cdot 3$ & 1.8 & $2 \cdot 7$ & 0.052 & $-0.287^{\star *}$ \\
\hline
\end{tabular}

FFM, fat-free mass; FM, fat mass; TEE, total energy expenditure; BW, body weight; PAL, physical activity level ( = TEE/BMR); PAEE, physical activity energy expenditure ( $=0.9 T E E-$ BMR); METs, metabolic equivalents. "Significant correlation with \%BF: * $P<0.05$, ${ }^{* *} P<0.01,{ }^{* \star *} P<0.001$.

Mean value was significantly different from that for the 1 st quartile: $\dagger P<0.05$, $\dagger \dagger P<0.01$.

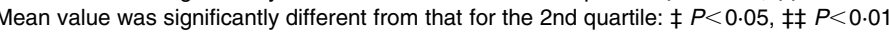

Mean value was significantly different from that for the 3rd quartile: $\S P<0.05$, $\S \S P<0.01$.

Subjects were categorised by quartile. There are twenty-five subjects in each quartile.

आ Because some variables in physical characteristics did not follow a normal distribution, Kruskal-Wallis analysis was used to compare the variables among quartiles, and the Mann-Whitney $U$ test was used for multiple comparisons. 
(a)

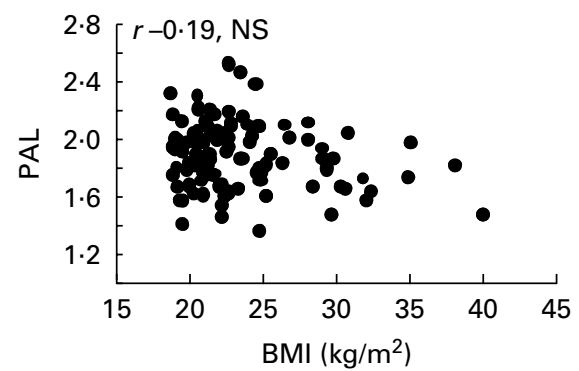

(b)

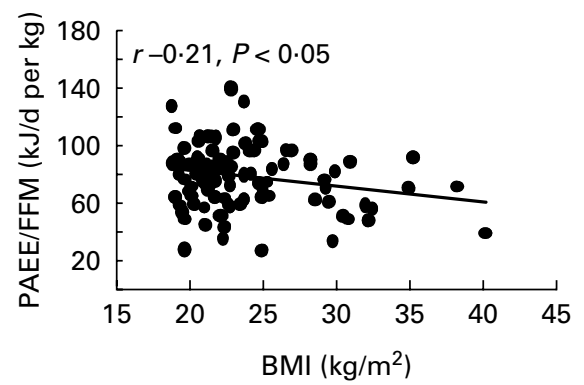

(c)

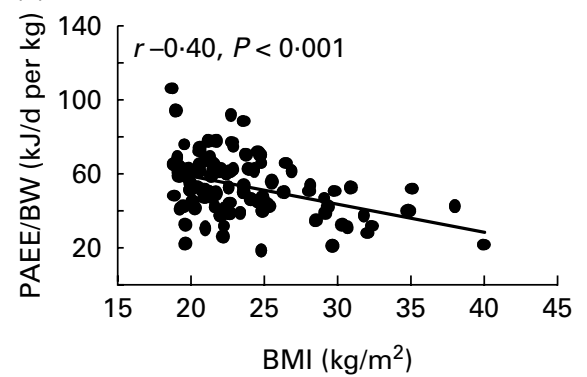

FFMI
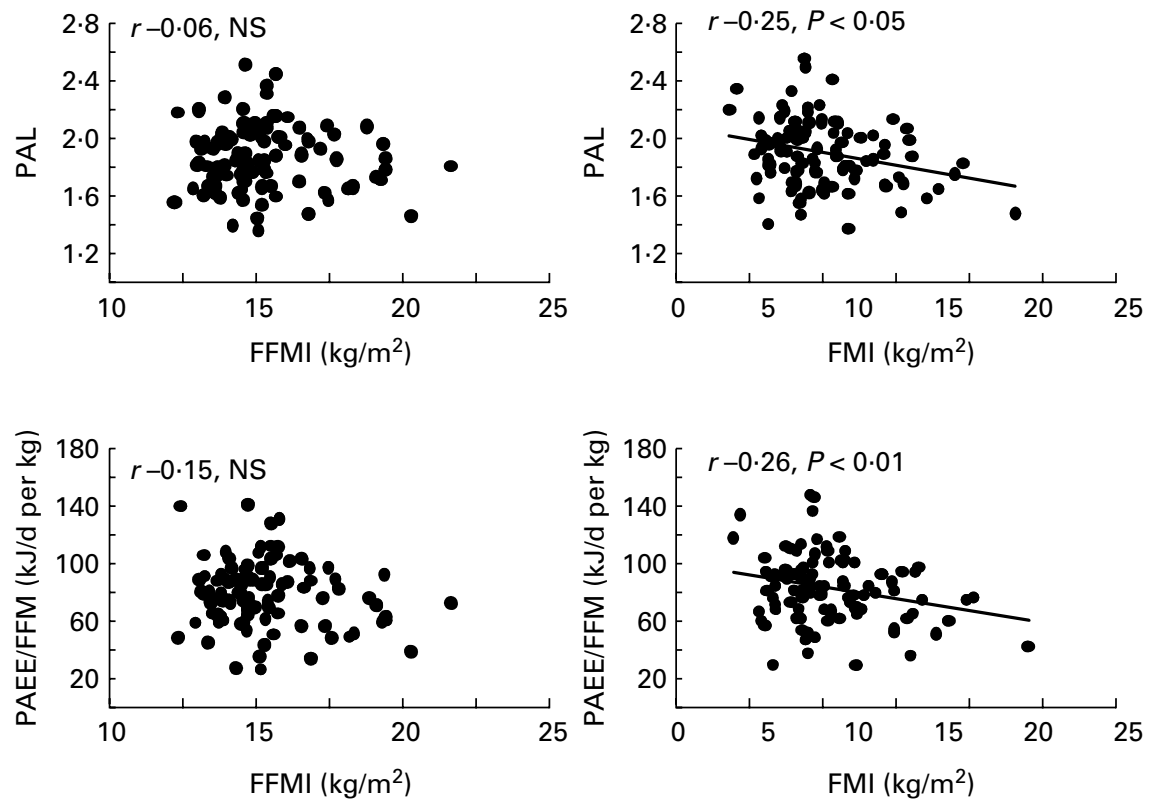

Fig. 2. Relationships between BMI, fat-free mass index (FFMI) or fat mass index (FMI) and physical activity level (PAL) (a), physical activity-related energy expen-

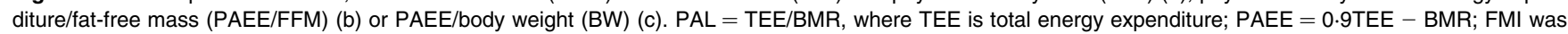
negatively associated with all physical activity variables obtained by the doubly-labelled water method.

negatively associated with BMI and FMI, but not with FFMI (Fig. 2).

In the accelerometry data, the step counts decreased in the 4th quartile of FMI (Table 3) and \%BF (Table 4), whereas there was no difference among quartiles of BMI (Table 1) and FFMI (Table 2). Time spent on moderate- or vigorous-intensity activity decreased in the 4th quartile of FMI, whereas it did not differ among quartiles of BMI, FFMI and \%BF. Time spent on light-intensity activity did not differ among quartiles of BMI, FFMI, FMI and \%BF.

\section{Discussion}

The principal finding in the present study was that only PAEE/ FFM and PAEE/BW assessed by the DLW method decreased among women in the highest quartile of BMI. On the other hand, women in the highest quartiles of FMI and \%BF obviously had a low level of physical activities assessed by both the DLW method and accelerometer. Particularly, women in the 3rd quartile of FMI or \%BF had lower PAEE/ BW even though their BMI was below $25 \mathrm{~kg} / \mathrm{m}^{2}$.
The average PAL of 1.88 in the participants of the present study was a little higher than that of 1.75 in the general population of Eastern or Western countries ${ }^{(7,16,23,24)}$. The average BMR in the present data was $88.3 \mathrm{~kJ} / \mathrm{d}$ per $\mathrm{kg} \mathrm{BW}$ for normal-weight women $\left(\mathrm{BMI}<25 \mathrm{~kg} / \mathrm{m}^{2}\right.$ ) and $76.2 \mathrm{~kJ} / \mathrm{d}$ per $\mathrm{kg}$ BW for overweight women $\left(\mathrm{BMI} \geq 25 \mathrm{~kg} / \mathrm{m}^{2}\right)$. These values were close to the average BMR of $88.8 \mathrm{~kJ} / \mathrm{d}$ per $\mathrm{kg}$ BW for Japanese normal-weight adult women ${ }^{(25)}$ and $74.9 \mathrm{~kJ} / \mathrm{d}$ per $\mathrm{kg}$ BW in Japanese overweight adult women ${ }^{(19)}$. Moreover, the range of PAL in the present study was $1 \cdot 36-2 \cdot 52$, which is within the PAL of the general population $^{(26)}$. The average daily steps of about 8500 for participants in the present study were also comparatively higher than the daily steps for Japanese adults women, who generally walk an average of 7215 steps/ $\mathrm{d}^{(27)}$.

The lack of a significant difference in PAL among BMI quartiles in the present study is consistent with most previous studies $^{(4-6)}$. In contrast, Tooze et al. ${ }^{(28)}$ demonstrated that PAL was lower in obese women $\left(B M I \geq 30 \mathrm{~kg} / \mathrm{m}^{2}\right)$ than in normal-weight women (BMI $<25 \mathrm{~kg} / \mathrm{m}^{2}$ ). However, they used an estimated RMR, but not a measured rate, so some errors in estimating PAL may be induced by the 
Table 5. Concordance of classification between BMI and fat mass index (FMI) or percentage body fat (\%BF)

(Percentages and number of subjects)

\begin{tabular}{|c|c|c|c|c|c|c|c|c|c|c|c|c|c|c|c|c|}
\hline \multirow{3}{*}{$\begin{array}{l}\text { Quartile*... }^{*} \\
\text { BMI quartile }\end{array}$} & \multicolumn{8}{|c|}{ FMI } & \multicolumn{8}{|c|}{$\% B F$} \\
\hline & \multicolumn{2}{|c|}{$1 \mathrm{st}$} & \multicolumn{2}{|c|}{ 2nd } & \multicolumn{2}{|c|}{$3 r d$} & \multicolumn{2}{|c|}{ 4th } & \multicolumn{2}{|c|}{$1 \mathrm{st}$} & \multicolumn{2}{|c|}{ 2nd } & \multicolumn{2}{|c|}{$3 r d$} & \multicolumn{2}{|c|}{ 4th } \\
\hline & $\%$ & $n$ & $\%$ & $n$ & $\%$ & $n$ & $\%$ & $n$ & $\%$ & $n$ & $\%$ & $n$ & $\%$ & $n$ & $\%$ & $n$ \\
\hline 1st (lowest) & 68 & 17 & 32 & 8 & 0 & 0 & 0 & 0 & 60 & 15 & 28 & 7 & 12 & 3 & 0 & 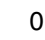 \\
\hline 2nd & 28 & 7 & 44 & 11 & 28 & 7 & 0 & 0 & 36 & 9 & 32 & 8 & 32 & 8 & 0 & 0 \\
\hline 3rd & 4 & 1 & 24 & 6 & 56 & 14 & 16 & 4 & 4 & 1 & 32 & 8 & 40 & 10 & 24 & 6 \\
\hline 4th (highest) & 0 & 0 & 0 & 0 & 16 & 4 & 84 & 21 & 0 & 0 & 8 & 2 & 16 & 4 & 76 & 19 \\
\hline
\end{tabular}

* There are twenty-five subjects in each quartile.

different accuracy of estimated RMR between lean and obese participants ${ }^{(19)}$.

Only PAEE/FFM and PAEE/BW decreased among women in the highest quartiles of BMI, whereas not only PAEE/FFM and PAEE/BW but also PAL apparently decreased in the highest quartile of FMI and \%BF. Based on the results of the concordance of classification between BMI and FMI or \%BF, most participants with a higher BMI have higher FM as well (Table 5). Thus, women in the highest quartile of BMI might be less active on the basis of PAEE when adjusting for body size. Contrary to the results of the present study, Snodgrass et al. ${ }^{(29)}$ reported that PAEE/BW was not different between lean and overweight women. However, lean and normalweight women in their study had much lower PAL (1.43 (SD $0 \cdot 21)$ ) and two of the seven women were underweight $\left(\mathrm{BMI}<18.5 \mathrm{~kg} / \mathrm{m}^{2}\right)$.

In contrast to the results of the decrease in PAEE/FFM and PAEE/BW among women in the highest quartile of BMI, there were no differences in PAEE/FFM and PAEE/BW among normal-weight women in the 1st to 3rd quartiles of BMI. Among participants in the 3rd quartile of BMI, the proportion of participants who are included in the 3rd quartile of FMI was only about half and the remaining spread to the other quartiles of FMI (Table 5). This phenomenon was similar to that of participants in the 2nd quartile of BMI. Thus, there appears to be a considerably large interindividual variability, especially for PAEE/FFM in normal-weight women who have a different distribution of FFM and FM at the same BMI.

The present study showed that TEE/BW was correlated with BMI, FMI or \% BF. However, the overcorrection of TEE when adjusted by BW should be cautiously interpreted, because BMR accounts for approximately $60 \%$ of TEE in an individual with a PAL of $1 \cdot 75$. On the other hand, in PAEE, which is not influenced by BMR, someone with a larger body mass needs more energy for an activity than someone with a smaller body mass. Thus, PAEE/BW may well reflect lower physical activity among women in the highest quartile of BMI. However, we could not exclude the possibility that PAEE/BW might be also adjusted excessively because there was a great difference in BW and FM between the 3rd and 4th quartile of BMI in the present study. However, among quartiles of FMI and \%BF, PAEE/BW was lower in the 3rd quartile than in the 1st or 2nd quartile, although it was not a great difference in BW between the 3rd quartile and the 1st or 2nd quartile. Therefore, lower PAEE/BW could well reflect the status of lower physical activity in women with higher BMI, especially with higher fat deposition, when FMI or \%BF was effectively used.

Schulz et l. $^{(7)}$ reported a high correlation between PAEE/ $\mathrm{BW}$ and $\% \mathrm{BF}$ in healthy adult women, thereby providing support for our data that PAEE/BW decreased from the 3rd quartiles of FMI and \%BF. Thus, PAEE/BW could be useful to understand daily physical activity, especially in normalweight women with higher fat deposition.

Step counts and the duration of physical activity of moderate or vigorous intensity assessed by accelerometry apparently decreased in the highest quartile of FMI, but not among quartiles of BMI and FFMI. Contrary to the present results of no difference in step counts and moderate or vigorous intensity among BMI quartiles, Levine et al. ${ }^{(30)}$ reported that the allocation of standing and ambulating during the day was lower in obese subjects than in lean subjects when using BMI cutpoints. This discrepancy may be due to the different range of PAL among populations. Levine et al. ${ }^{(30)}$ recruited both lean and obese individuals from among 'couch potato' subjects, all of whom were sedentary. The populations of the present study were free-living Japanese adult women with a wide PAL range from sedentary to active.

In a longitudinal study using the DLW method in adult women, Schoeller et al. ${ }^{(31)}$ demonstrated that increases in weight were lower in active women with a PAL above 1.75. The present study did not attempt to determine a threshold of daily physical activity that is required to have a normal FMI, \%BF or BMI due to the limited number of study subjects and the proportion of obese individuals in the present dataset. Another reason was that there were no definite cut-offs for FMI and \%BF. Because the present study apparently showed a good relationship between FM (FMI or \%BF) and various physical activities, further study is warranted to examine the threshold of daily physical activity that is required to suppress fat accumulation.

The BMI cut-off point is used as the standard for a classification of obesity. On the other hand, Bigaard et al. suggested that FMI was also an independent predictor of all-cause mortality in their epidemiological study ${ }^{(32)}$. They revealed that an excess of approximately $10 \mathrm{~kg} / \mathrm{m}^{2}$ of FMI value was associated with considerably increased mortality. The present study showed that Japanese adult women with an average FMI of $12.6 \mathrm{~kg} / \mathrm{m}^{2}$ were less active than those with a below-average FMI of $8.6 \mathrm{~kg} / \mathrm{m}^{2}$. Therefore, we consider that an increase in 
PAL may decrease FMI, leading to a decrease in risk of all-cause mortality.

The present study has the following limitations: first, the FFM hydration was assumed as 0.732 for all participants equally $^{(14)}$, so some errors in estimating FFM may be induced by the different levels of obesity. Second, the present results were drawn from a cross-sectional design. Therefore, we were not able to infer a cause-effect relationship between an inactive lifestyle and obesity. Observational or intervention studies with longitudinal design are needed to evaluate the effect of inactivity on the development of obesity for adult women. However, the main purpose of the present study was to investigate the relationship between daily physical activity and body size or body composition. Moreover, the present study provided the results only for Japanese adult women, but not for men or children.

In conclusion, Japanese adult women with larger BMI had lower PAEE adjusted by FFM or BW. Especially, Japanese adult women with higher fat deposition were apparently less active, on the basis of not only PAEE but also the physical activity of moderate or vigorous intensity. The present data suggest that the relationship between obesity and daily physical activities should be discussed using not only BMI but also FMI or \%BF.

\section{Acknowledgements}

The present study was performed as part of the Health and Labor Sciences Research Grants (Comprehensive Research on Cardiovascular and Life-style Related Diseases) from the Ministry of Health, Labour and Welfare of Japan. We thank the staff of the National Institute of Health and Nutrition for their kind cooperation.

The authors' responsibilities were as follows: interpretation of the data and writing the manuscript, J. P., K. I-T. and S. T.; DLW analysis, J. P., K. I-T. and Y. H.; conception, design, and conducting of DLW studies and obtaining funding, K. I-T., S. T. and I. T.; aiding in designing the manuscript and providing critical revision, Y. H. and K. O.; designing and conducting the Saku Control Obesity Program, K. I-T., S. T., S. W., M. M., A. M. and N. A.; obtaining funding for Saku Control Obesity Program, S. W.

The authors have no relevant financial interest in this article.

\section{References}

1. Schoeller DA (2001) The importance of clinical research: the role of thermogenesis in human obesity. Am J Clin Nutr $\mathbf{7 3}$, $511-516$

2. Schoeller DA (2008) Insights into energy balance from doubly labeled water. Int J Obes (Lond) 32, S72-S75.

3. Ferro-Luzzi A \& Martino L (1996) Obesity and physical activity. Ciba Found Symp 201, 207-221.

4. Prentice AM, Black AE, Coward WA, et al. (1996) Energy expenditure in overweight and obese adults in affluent societies: an analysis of 319 doubly-labeled water measurements. Eur J Clin Nutr 50, 93-97.

5. Butte NF, Treuth MS, Mehta NR, et al. (2003) Energy requirements of women of reproductive age. Am J Clin Nutr 77, 630-638.
6. Das SK, Saltzman E, McCrory MA, et al. (2004) Energy expenditure is very high in extremely obese women. J Nutr 134, 1412-1416.

7. Schulz LO \& Schoeller DA (1994) A compilation of total daily energy expenditures and body weights in healthy adults. Am J Clin Nutr 60, 676-681.

8. Yao M, McCrory MA, Ma G, et al. (2003) Relative influence of diet and physical activity on body composition in urban Chinese adults. Am J Clin Nutr 77, 1409-1416.

9. Tudor-Locke C, Ainsworth BE, Whitt MC, et al. (2001) The relationship between pedometer-determined ambulatory activity and body composition variables. Int $J$ Obes Relat Metab Disord 25, 1571-1578.

10. Mitsui T, Shimaoka K, Tsuzuku S, et al. (2008) Pedometerdetermined physical activity and indicators of health in Japanese adults. J Physiol Anthropol 27, 179-184.

11. Sternfeld B, Bhat AK, Wang H, et al. (2005) Menopause, physical activity, and body composition/fat distribution in midlife women. Med Sci Sports Exerc 37, 1195-1202.

12. Watanabe S, Morita A, Aiba N, et al. (2008) Study design of the Saku Control Obesity Program (SCOP). Anti-Aging Med 5, 13-16.

13. Sakai S, Yanagibori R \& Amano K (1998) Self-administered diet history questionnaire developed for health education: a relative validation of the test-version by comparison with 3-day diet record in women. J Epidemiol 8, 203-215.

14. Pace N \& Rathbun EN (1945) Studies on body composition, III: The body water and chemically combined nitrogen content in relation to fat content. J Biol Chem 158, 685-691.

15. Weir JBV (1949) New methods for calculating metabolic rate with special reference to protein metabolism. J Physiol 109, 1-9.

16. Black AE, Prentice AM \& Coward WA (1986) Use of food quotients to predict respiratory quotients for the doubly labeled water method of measuring energy expenditure. Hum Nutr Clin Nutr 40C, 381-391.

17. Jones PJH \& Leitch CA (1993) Validation of doubly labeled water for measurement of calorie expenditure in collegiate swimmers. J Appl Physiol 74, 2909-2914.

18. Reed GW \& Hill JO (1996) Measuring the thermic effect of food. Am J Clin Nutr 63, 164-169.

19. Tanaka S, Ohkawara K, Ishikawa-Takata K, et al. (2008) Accuracy of predictive equations for basal metabolic rate and the contribution of abdominal fat distribution to basal metabolic rate in obese Japanese people. Anti-Aging Med 5, 17-21.

20. Kumahara H, Schutz Y, Ayabe M, et al. (2004) The use of uniaxial accelerometry for the assessment of physical-activityrelated energy expenditure: a validation study against whole-body indirect calorimetry. Br J Nutr 91, 235-243.

21. McClain JJ, Craig CL, Sisson SB, et al. (2007) Comparison of Lifecorder EX and ActiGraph accelerometers under freeliving conditions. Appl Physiol Nutr Metab 32, 753-761.

22. Schneider PL, Crouter SE \& Bassett DR (2004) Pedometer measures of free-living physical activity: comparison of 13 models. Med Sci Sports Exerc 36, 331-335.

23. Westerterp KR (1999) Obesity and physical activity. Int J Obes (Lond) 23, Suppl. 1, 59-64.

24. Ishikawa-Takata K, Tabata I, Sasaki S, et al. (2008) Physical activity level in healthy free-living Japanese estimated by doubly-labeled water method and International Physical Activity Questionnaire. Eur J Clin Nutr 62, 885-891.

25. Ministry of Health, Labour and Welfare of Japan (2010) Dietary Reference Intakes for Japanese, 2010 (in Japanese). Tokyo: Daiichi Shuppan.

26. Westerterp KR (1998) Alterations in energy balance with exercise. Am J Clin Nutr 68, 970S-974S. 
27. Ministry of Health, Labour and Welfare of Japan (2006) The National Health and Nutrition Survey in Japan, 2006 (in Japanese). Tokyo: Daiichi Shuppan.

28. Tooze JA, Schoeller DA, Subar AF, et al. (2007) Total daily energy expenditure among middle-aged men and women: the OPEN study. Am J Clin Nutr 86, 382-387.

29. Snodgrass JJ, Leonard WR, Tarskaia LA, et al. (2006) Total energy expenditure in the Yakut (Sakha) of Siberia as measured by the doubly labeled water method. Am J Clin Nutr 84, 798-806.
30. Levine JA, Lanningham-Foster LM, McCrady SK, et al. (2005) Interindividual variation in posture allocation: possible role in human obesity. Science 307, 584-586.

31. Schoeller DA, Shay K \& Kushner RF (1997) How much physical activity is needed to minimize weight gain in previously obese women? Am J Clin Nutr 66, 551-556.

32. Bigaard J, Frederiksen K, Tjønneland A, et al. (2004) Body fat and fat-free mass and all-cause mortality. Obes Res 12, 1042-1049. 